\title{
Development of green insulation boards from kenaf fibres part 2: characterizations of thermal and water absorption
}

\begin{abstract}
The aim of this study was to investigate the thermal performance, water absorption and dimension stability against water of the green insulation boards. The results show that the thermal conductivity decreased with increasing fibre contents and reached its minimum value (0.0535 w/mk) for the 60/40 kenaf / PU weight \%. Contrarily, thermal resistance increased with increasing fibres contents, up to its maximum value $(0.09 \mathrm{k} . \mathrm{m} 2 / \mathrm{w})$ for the $60 / 40 \mathrm{kenaf} /$ PU weight $\%$. The minimum water absorption percentage and thickness swelling were recorded at a weight of $50 \%$ kenaf fibres. The effects of the alkaline treatment were significant enough to increase the thermal conductivity.
\end{abstract}

Keyword: Biocomposites; Laminates; Natural materials 